\title{
Open Science cannot Succeed without Open Peer Review
}

\section{Giannis Tsakonas}

\author{
Library \& Information Center, University of Patras, Greece \\ gtsak@upatras.gr, orcid.org/0000-0002-8786-9440
}

Open Science principles have been a critical driver for change in scholarly communication. Opening up research publications has led to encouraging rates of growth of Open Access but it has now become evident that true and system-wide change will only come if all the publishing components are open, including those at the production stages. We are seeing a growing demand to know more about how research papers qualify for publication and if we wish to engender more trust in scholarly outputs - trust within the scholarly communication community and trust of the wider public - we should listen carefully and seek to act on these demands. However, when an entire system has been fixed in its ways for many years, it is not easy to shake things up. In short, while it may be reasonable to ask for changes, not all of these will be welcomed or be embraced.

Peer review is one of these publishing components. Regardless of the types and forms in each publication venue, it supposedly ensures that the intellectual work of an author has been checked, improved and qualified for publication after constructive dialogue among all participants; the author, the editor and the reviewers. We recognise it as an important process that validates the quality of a publication, which can be trusted by the reader and - as trusted - will be used in solving research problems and sometimes underpin changes in public policy. While the principles of peer review are still valid, in an Open Science system the current closed and entirely opaque operation of this system seems obsolete and even vicious. Masked by anonymisation and based on linearity this very regulated dialogue serves largely the prestige of publishing venues and individuals. Changes are needed, but we should acknowledge beforehand that changing peer review is a challenging exercise. The issues around peer review are complex, requiring alternative management of both the processes and the actors.

This work is licensed under a Creative Commons Attribution 4.0 International License

Uopen Journals | http://liberquarterly.eu/ | DOI: 10.53377/lq.11114 


\section{Processes}

We could classify two main issues regarding processes, both very challenging: the transparency and the promptness of peer reviewing. Open peer review has started appearing in many publishing venues. It requires that all actors are known to each other and that the contributions are known to the readers as well. In some cases, the authors can propose the reviewers themselves and an editor facilitates reviewing in the best interest of the manuscript. The F1000 platform has been pivotal in giving an example of a functional system that supports these conditions in an accessible and readable way. It now gives the opportunity to the European Commission to support its Open Science practices by providing the Open Research Platform ${ }^{1}$ with the same characteristics. As the manuscript evolves to take its final form, the reports of the reviewers accompany each of its versions.

Publishing a work months after the submission date (let alone the access issues we sometimes see after publication) risks failure to solve in a timely way the problems addressed by that research. Quite often the reasons for long delays are not easily explained, while the world of academic Twitter associates these with the prestige of the publishing venues. Frustration about such delays fuels the economy of predatory journals, the delusional world of quick and easy publication that can be harmful to early career researchers or marginalised authors.

Openness does not come only in the form of transparency, but also in the form of transversal publication modes. Where a manuscript has been rejected by one publisher, we are seeing limited adoption of the routine migration of reviews to the next (potential) publishing venue. Even where this does happen, we see difficulties arising between organisational and technical silos that continue to obscure the rationale of a decision not to publish. Advances in the technology propose a new scholarly communication system that embraces repository infrastructures and gives the opportunity to start the review prior to the formal stages or to open the review to many other readers; not necessarily peers. The case of PreReview, ${ }^{2}$ which flourished when the pandemic research was rapidly opened up, is an apt example of opening preprints for reviewing, but there are several other initiatives that link preprint repositories to journals, creating the conditions for overlay journals. ${ }^{3}$ Such links further consolidate the status of Open Science, as they acknowledge repository services as active scholarly components and not mere passive archives, engage 
more actors in the reviewing process and save time and effort by channeling openly the intellectual work of reviewers. Whereas there are concerns about the effect of these new approaches ${ }_{,}^{4}$ it has to be noted that these do not substitute the minimum controls for quality, nor do they make publications appear as something else (e.g. a fully published work). These new platforms are progressing constructive dialogue among all parties, they ensure that reviews and responses address the key issues of a manuscript, they minimise any kind of bias and they facilitate reproducibility. In a world where everyone can (and does) openly discuss all kinds of issues, these new platforms create opportunity to expand the dialogue even after publication, with tools for building a robust and clear review narrative over a period of time and representing multiple voices.

\section{Actors}

Reviewing is an inherent characteristic of academia and research, but it is often unacknowledged. Reviewers need access to literature and they each provide a service that requires time, expertise and careful consideration. While we have seen the introduction of systems that acknowledge these tasks, these do not seem to serve a wider purpose, because they address the issue on the individuals' level; they remain decontextualised and serve only the robustness of the host platform. The lack of policies that record the reviewing service, recognise it and embed it in the research assessment, is stalling the actual acknowledgement and by extension the overview of a researcher's contribution. Advocates of Open Access raise an important question: why are peer reviewers not compensated, particularly given that their reviews are often conducted to support for-profit publishers? These advocates highlight the unfairness of a system that is not rewarding all the time, effort and expert care and they decide - consistent with their Open Access ethos - to serve their community through other venues, such as Diamond OA journals, which are largely dependent on this voluntary stance and/or repositories.

Recruiting competent reviewers, with productive outcomes, is reported as one of the main editorial problems. There are signs of fatigue among reviewers, but there are also growing calls on academic time from other areas, such as research funding proposals. By choosing or being forced to stay with a controlled 
number of experts, the pathogenies -all the causes underlying this terminally malfunctioning system- are inevitable. Eventually we see the inevitable results: stretching the one thing that cannot be stretched, which is time, and/or delivering poorer quality reviews that are masked by the anonymous peer review practice. This closed peer reviewer management practice also raises important questions about how we can ever understand and measure the diversity and representativeness of the established peer reviewer community.

Not all innovations of Open Science seem ready to be embedded in the scholarly communication cycle. However, they open the discussion about the invitation of more reviewers, possibly not peer, but within a range of expertise, in order to have a wider and an alternative view. Braver communities, especially in fields where citizen science thrives, can adopt systems that even after the publication can propose points of interest for further improving the manuscript. This symbiosis remains to be assessed and we need to see how reviewing can be enhanced or indeed validated by Artificial Intelligence techniques, such as natural language processing or sentiment analysis, in classifying, i.e. accept/reject, scientific contributions.

\section{The Role of Research Libraries}

Libraries can play a critical role in disseminating novel practices that help to evolve the peer review process. By identifying malpractices, they can protect the research community from contagious phenomena, like predatory publishing, and foster greater understanding of Open Access research publishing. Raising awareness and engaging with the communities to receive their concerns is also vital, while libraries with a certain level of infrastructure can support them by registering assets, such as data, protocols and so on, that enhance reproducibility. Libraries that have an active role in OA publishing - in journals or monographs platforms, have an opportunity to trailblaze policies of innovative peer review and cultivate another perspective with their editorial teams. In a recent workshop ${ }^{5}$ of LIBER $^{6}$ and Open Research Europe, quite a few questions were expressed about how open peer review can be facilitated in other types of publication, such as monographs, especially in the fields where the journal article is not the typical unit of contribution. These questions require the vital space to be heard and while they might seem minor in the face of other more impending issues, posing them alone can light the touch-paper of much bigger change. 


\section{Conclusion}

The innovations of Open Science question the typical profile of peer review that has been frozen for decades. Responding to the social demand for openness in academia, one hopes that open peer review can be installed as an inherent property of modernised publishing systems. While the desire is to have Open Science as the new norm for all research, one must remain pragmatic given that the paradigm might not entirely prevail in the forthcoming years. We should not expect that all publishing venues will proceed in such an open way, but there should be options for the communities that wish to do so. However, it is necessary that these open options are considered and rewarded by all stakeholders in order to have a fair and sustainable system for all.

Many believe that peer review should embrace the technical advancements and the opportunities they present, as well as be part of the cultural change in institutions and policies that Open Science has introduced. But innovative peer review pushes scholarly communication to new limits by making the status of scientific publication more dynamic. The versions of a manuscript that has been changed after stages of revision, all cited with permanent identifiers to enable retrieval, is a step forward from the paradigm of a static instance of an intellectual work.

Many of these developments have happened in a short time and it might be early to have a full view of the successful - and less successful - interventions, but peer review is gaining attention in the world of Open Science. Where human factors and/or conservative establishments have created enclaves of prejudice and discrimination, Open Science is proposing new ones that are more transparent and justly recognise the critical role of reviews in research publishing.

\section{Notes}

\footnotetext{
${ }^{1}$ https://f1000research.com/about - F1000Research is an Open Research publishing platform for scientists, scholars and clinicians offering rapid publication of articles and other research outputs without editorial bias. All articles benefit from transparent peer review and editorial guidance on making all source data openly available.
} 
${ }^{2}$ https://prereview.org.

${ }^{3}$ Overlay journals are publishing initiatives that work together with repository services, but on another level, another "layer". Overlaid publishing has various adaptations, but in principle -and in relation to the matter of peer review-it reverses the process of "first review, then publish" to "first publish, then review". The concept first appeared in a paper of Paul Ginsparg in 1997 (https:/ / doi.org/10.1300/ J123v30n03 13).

${ }^{4}$ While more than two decades old arguments in this opinion paper (https:// doi.org/10.1038/6295) still echo in our days, the effects of "publication urge" on repositories and journals and the expediting of review can be summarised at https:// www.nature.com/articles/d41586-020-01394-6.

${ }^{5}$ https:/ / libereurope.eu/mec-events/innovative-peer-review-for-research-librariesworkshop-at-open-science-fair/.

${ }^{6}$ https:/ / libereurope.eu - LIBER (Association of European Research Libraries) is the voice of Europe's research library community. 